\title{
Enhancement of Fatigue Properties in Sensor Element by Surface Modification
}

\author{
Kohei Takeda* \\ Department of Mechanical Engineering, Aichi Institute of Technology, \\ 1247 Yachigusa, Yakusa-cho, Toyota City, Aichi 470-0356, Japan
}

(Received March 31, 2020; accepted July 14, 2020)

Keywords: sensor element, shape memory alloy, superelasticity, ultrasonic shot peening, fatigue

The fatigue property of TiNi shape memory alloy (SMA) is one of the important issues for its use as a sensor element. In this study, the fatigue life of TiNi SMA was improved by ultrasonic shot peening (USP), which can provide the same or a better effect than general shot peening. The effects of the residual stress, surface roughness, and fatigue crack initiation position on the fatigue life of TiNi SMA wires treated by USP were investigated. The results obtained are summarized as follows. (1) The value of the upper stress plateau under the loading process is increased by USP. The increase in stress is almost proportional to the coverage. (2) In the rotating-bending fatigue test, the fatigue life is improved by USP with coverages of 200, 1000, and $2000 \%$. (3) The higher the coverage, the higher the percentage of fatigue cracks that appear from the specimen surface in USP specimens.

\section{Introduction}

Shape memory alloys (SMAs) have drawn interest as intelligent materials and have attracted worldwide attention as sensor elements, ${ }^{(1,2)}$ the latter because of their unique properties of a shape memory effect and superelasticity. ${ }^{(3-5)}$ Among the SMAs, TiNi SMA has a longer fatigue life, greater recovery strain, and better corrosion resistance than other SMAs. ${ }^{(6-8)}$ However, in order to increase the practical use of TiNi SMA as a sensor element, requirements such as a long fatigue life, high corrosion resistance, and high wear resistance must be satisfied. A TiNi SMA sensor element is usually a thin wire due to the thermal response, and tensile or rotatingbending fatigue tests have been performed to evaluate the fatigue properties of TiNi SMA wires. ${ }^{(9-12)}$

On the other hand, it is well known that fatigue properties are improved by the surface modification of metallic materials such as by shot peening and nitriding. Although there are reports on the crystal structure of SMA subjected to surface modification, there are few reports on the fatigue life. In previous research, the improvement of fatigue properties was confirmed when ultrasonic shot peening (USP) was applied to a TiNi SMA tape. ${ }^{(13)}$ However, fatigue cracks were initiated at positions on the surface not treated by USP. Although the flat surface

*Corresponding author: e-mail: k-takeda@aitech.ac.jp

https://doi.org/10.18494/SAM.2020.2893 
of the tape was treated with USP, the side surface was not treated owing to the thickness of the specimen being less than the diameter of the shot medium. Therefore, the entire surface of a TiNi SMA wire should be treated by USP while rotating the specimen. In this study, the effects of USP on the fatigue, tensile deformation, and surface properties of TiNi SMA wires were investigated.

\section{Materials and Methods}

\subsection{Material}

The material used in this study was TiNi SMA wire manufactured by Furukawa Techno Material Co., Ltd., which shows superelasticity at room temperature. The composition ratio of the TiNi SMA was Ti-50.94 at $\% \mathrm{Ni}$, where $\mathrm{Ti}$ and $\mathrm{Ni}$ are almost equiatomic. The specimens used in the tensile test and the rotating bending fatigue test were $1 \mathrm{~mm}$ in diameter and $140 \mathrm{~mm}$ in length. USP was applied to a region of $40 \mathrm{~mm}$ near the longitudinal center of the specimen (about $70 \mathrm{~mm}$ from one end). The reverse transformation start temperature $A_{s}$ was $251 \mathrm{~K}$ and the reverse transformation finish temperature $A_{f}$ was $300 \mathrm{~K}$.

\subsection{Experimental apparatus and methods}

TiNi SMA wires were treated by USP to elucidate the effect of USP on the fatigue life. USP can provide the same or a better effect and lower surface roughness than general shot peening. ${ }^{(14)}$ A USP device manufactured by SONATS was used for the USP treatment. As the conditions for USP treatment, the coverages were 200,1000, 2000, and 4000\%, and the material of the shot medium was SUJ2 steel of $0.8 \mathrm{~mm}$ diameter. Figure 1 shows a schematic diagram of USP treatment. Indentations could not be clearly observed on the USP-treated specimen surface. The USP coverage was determined by using an Almen strip as follows: (1) Measure the time of indentation on the entire surface of the Almen strip and set this value to $100 \%$ coverage. (2) When the coverage is $2000 \%$, apply 20 times the time for $100 \%$ coverage of the Almen strip to the specimen. The specimen was rotated at $12 \mathrm{rpm}$ in the chamber of

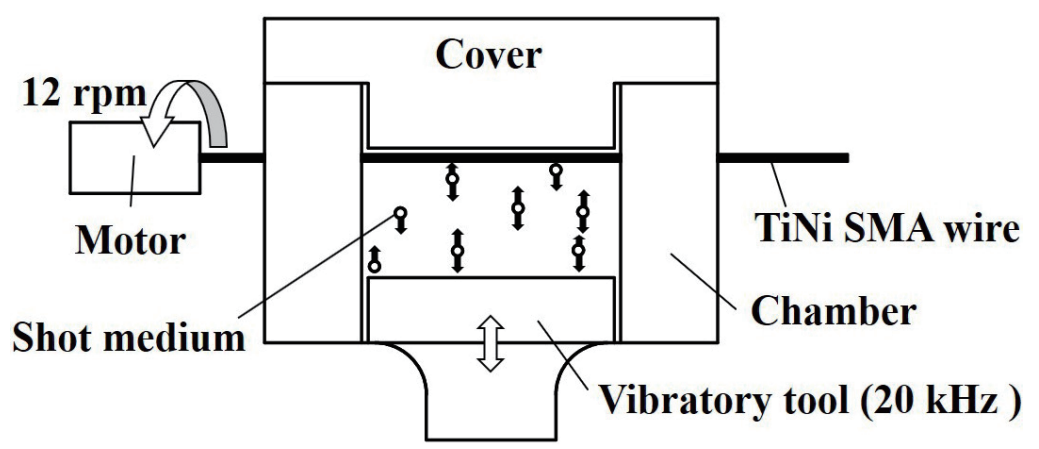

Fig. 1. Schematic diagram of USP treatment. 
the device to apply USP to the entire surface. In the tensile test, an extensometer with a gauge length of $20 \mathrm{~mm}$ was used for displacement measurement. The strain $\varepsilon$ was determined by the nominal strain. Tensile tests were performed at room temperature in air under a strain rate of $d \varepsilon / d t=1 \times 10^{-4} \mathrm{~s}^{-1}$. The residual stress was measured on the (211) plane of the austenitic phase by X-ray diffraction using the parallel tilt method with respect to the axial direction of the specimen. Table 1 shows the measurement conditions of the X-ray diffraction. In the fatigue test, a rotating-bending fatigue test machine was used. The test was performed in air at room temperature. The repetition frequency was $2.66 \mathrm{~Hz}(160 \mathrm{cpm})$. The bending strain amplitude was the maximum strain at the breaking point of the specimen in the rotating-bending fatigue test. The maximum strain on the specimen surface was determined by the radius of curvature at the breaking point. A scanning electron microscope (SEM) was used to observe the fracture surface of each specimen. A VK-X200 laser microscope manufactured by Keyence Corporation was used to measure the surface roughness of the specimen.

\section{Experimental Results and Discussion}

\subsection{Tensile deformation property}

The stress-strain curves obtained by the tensile test are shown in Fig. 2. The stress-strain curves show a hysteresis loop during the loading and unloading processes, and superelasticity, i.e., the recovery of the strain upon unloading, was observed under all conditions. The value of

Table 1

Measurement conditions of the X-ray diffraction.

\begin{tabular}{lc}
\hline X-ray radiation & $\mathrm{CrK} \alpha$ \\
Measurement method & Iso-inclination method \\
X-ray-irradiated diameter & $\phi 0.5 \mathrm{~mm}$ \\
Diffraction angle $2 \theta$ & $138.6 \mathrm{deg}$ \\
Diffractive plane & 211 \\
\hline
\end{tabular}

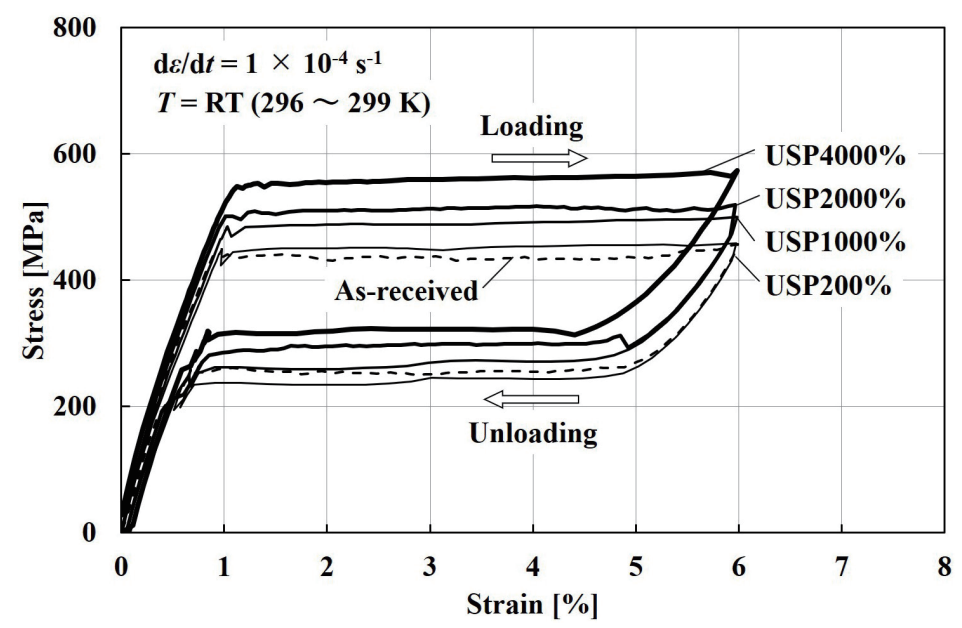

Fig. 2. Stress-strain curves of USP specimens and as-received material obtained by the tensile test. 
the upper stress plateau of the USP-treated specimens was higher than that of the as-received specimen, and it increased with increasing coverage. This increase in stress may have been caused by the increase in the stress required for the martensitic transformation due to the compressive residual stress generated by the USP. The increase in stress was $16 \mathrm{MPa}$ for USP $200 \%, 56 \mathrm{MPa}$ for USP $1000 \%$, $74 \mathrm{MPa}$ for USP2000\%, and $123 \mathrm{MPa}$ for USP $4000 \%$ and for the as-received material. The increase in stress upon USP was almost proportional to the coverage.

\subsection{Residual stress}

The relationship between the residual stress and the depth from the surface obtained by X-ray diffraction is shown in Fig. 3. In the USP-treated specimens, the compressive residual stress is higher in the region closer to the surface. Also, the larger the coverage at the same depth, the higher the value of the compressive residual stress. In the cases of USP $200 \%$, USP $1000 \%$, and USP $2000 \%$, the difference from the as-received material became smaller deeper below the surface, and the same residual stress was obtained at a depth of about $80 \mu \mathrm{m}$. In the cases of USP2000\% and USP4000\%, the residual stress could not be clearly measured near the surface, possibly because the surface layer became amorphous upon USP.

\subsection{Rotating-bending fatigue life}

The relationships between the bending strain amplitude and the number of cycles to failure of the TiNi SMA wires obtained by the rotating-bending fatigue test at room temperature are shown in Fig. 4. As can be seen, the fatigue life becomes longer when USP is applied, especially at a smaller bending strain amplitude. However, the fatigue life of USP $4000 \%$ is almost equal to that of the as-received material. In general, if compressive residual stress is applied, the crack initiation is suppressed and the fatigue life increases. However, in Fig. 4, the fatigue life of USP $4000 \%$ is shortest even at a higher compressive residual stress. The shorter

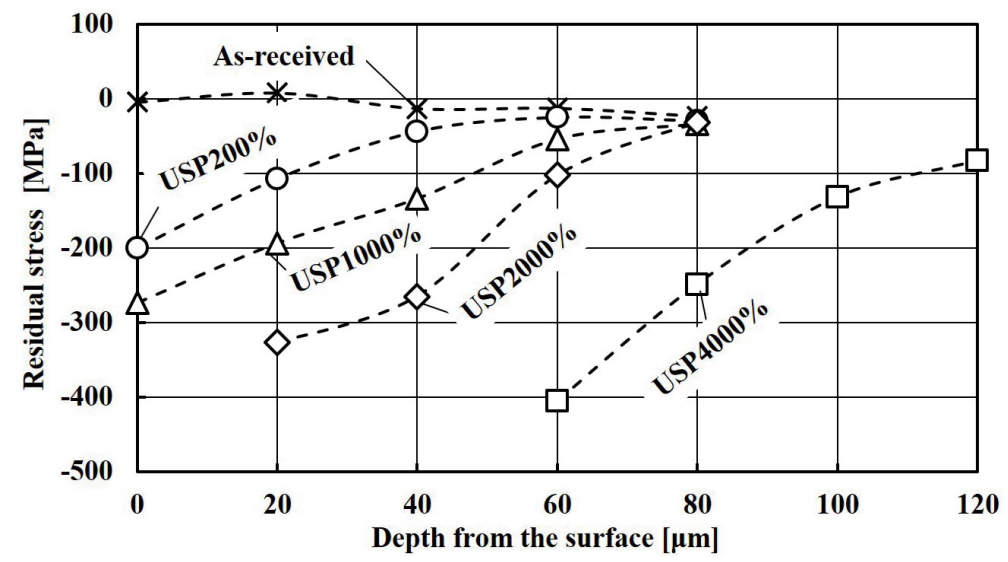

Fig. 3. Relationship between the residual stress and the depth from the surface. 


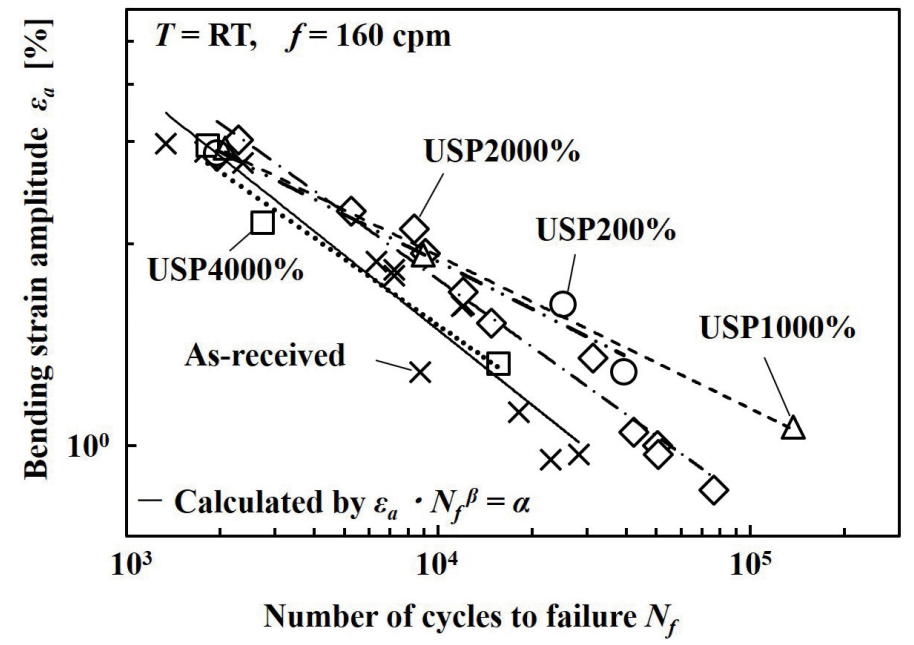

Fig. 4. Relationships between bending strain amplitude and number of cycles to failure of the TiNi SMA wire obtained by the rotating-bending fatigue test.

fatigue life of USP $4000 \%$ than the other USP-treated specimens may have been caused by the surface roughness and the amorphous layer near the surface.

The fatigue life curves shown on logarithmic scales are almost perfectly expressed by straight lines for all materials. The relationship can therefore be expressed by the power function inset in Fig. 4. The overall trends are well approximated by the lines.

\subsection{Surface roughness}

The relationships between the arithmetic mean roughness $R a$ in the axial direction of the specimen and the USP processing condition are shown in Fig. 5. It is well known that the surface roughness of a material generally affects its fatigue life; if the surface is rough, the fatigue life will be shorter. ${ }^{(15)}$ The roughness of the USP specimens is larger than that of the as-received material. Also, except for USP200\%, the higher the coverage of USP, the larger the roughness. The cause of the increased surface roughness in the case of USP $200 \%$ will be examined in a future study. On the other hand, although the roughness of USP $200 \%$ is the largest, the fatigue life is longer than those of the other USP specimens as shown in Fig. 4. This is due to the location of fatigue crack initiation, as discussed in the next section.

\subsection{Fatigue fracture surface}

Images of the fatigue fracture surface of the USP200\% and USP2000\% TiNi SMA wires obtained by SEM are shown in Figs. 6 and 7, respectively, where (a) shows an image of the entire fracture surface and (b) shows an enlarged image at the fatigue crack initiation position. The point $F_{c}$ in each image indicates the fatigue crack initiation position, and the broken white line indicates the fatigue crack propagation area. As can be seen in Fig. 6, in the case of 


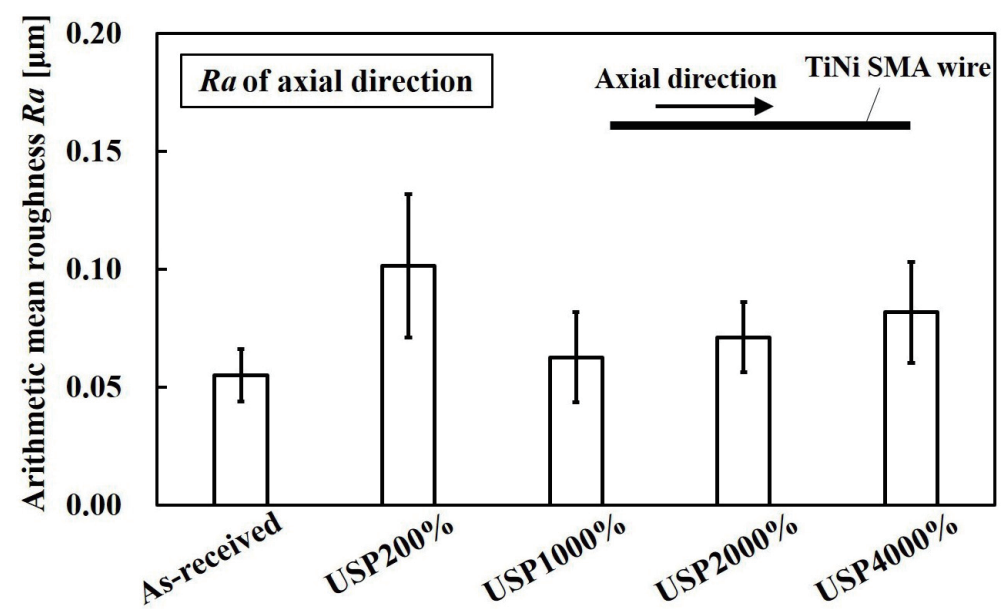

Fig. 5. Relationships between the arithmetic mean roughness $R a$ in the axial direction of the specimen and the USP processing condition.

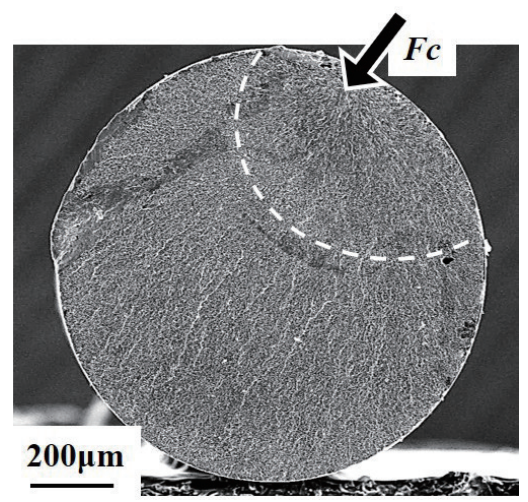

(a)

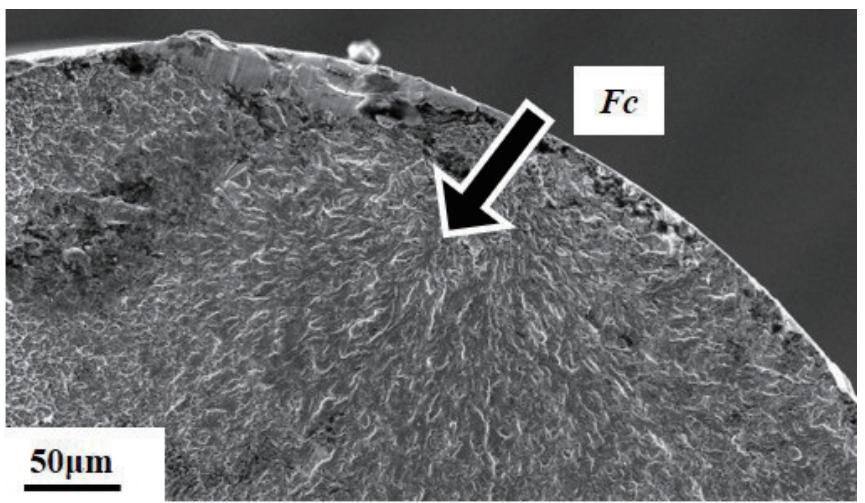

(b)

Fig. 6. SEM images of the fracture surface of USP200\%. (a) Whole fracture surface. (b) Enlarged image at crack initiation part.

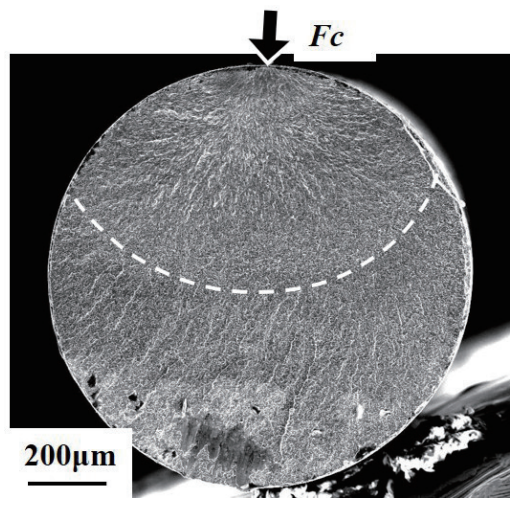

(a)

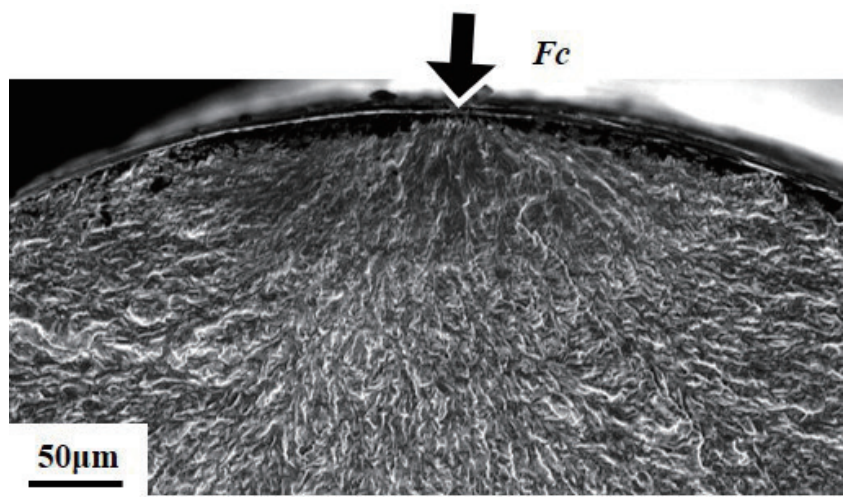

(b)

Fig. 7. SEM images of the fracture surface of USP2000\%. (a) Whole fracture surface. (b) Enlarged image at crack initiation part. 
Table 2

Number of fatigue crack initiation points in each specimen.

\begin{tabular}{|c|c|c|c|}
\hline USP condition & Surface & Internal & $\begin{array}{l}\text { Percentage of specimen } \\
\text { with internal crack (\%) }\end{array}$ \\
\hline As-received material & 12 & 0 & 0 \\
\hline USP $200 \%$ & 0 & 3 & 100 \\
\hline USP $1000 \%$ & 1 & 2 & 67 \\
\hline USP $2000 \%$ & 6 & 6 & 50 \\
\hline USP $4000 \%$ & 2 & 1 & 33 \\
\hline
\end{tabular}

USP $200 \%$, fatigue cracks occur from the inside near the specimen surface. On the other hand, cracks appear from the specimen surface in the case of USP2000\% as shown in Fig. 7. These behaviors are due to the surface roughness. Although the surface roughness of USP $200 \%$ is higher than that of the other USP specimens, the fatigue crack is generated from the inside owing to the compressive residual stress at the surface, resulting in a longer fatigue life.

Table 2 shows the number of locations of fatigue crack initiation in each specimen on which the fatigue test was performed and the percentage of specimen with internal crack. As can be seen in Table 2, all the fatigue crack initiation positions of the as-received material are on the specimen surface. In the USP specimens, the larger the coverage, the higher the ratio of fatigue cracks that initiate from the specimen surface. Hence, if the coverage is large, the surface roughness increases. Thus, the fatigue life becomes shorter. The internal fatigue cracking in USP $200 \%$ with a long fatigue life may have been caused by the compressive residual stress because of the absence of an amorphous layer at the specimen surface. These observations will be examined in detail in future work.

\section{Conclusions}

The effect of USP on the fatigue properties and tensile deformation characteristics of TiNi SMA wires was investigated. Furthermore, from the observation of the fatigue fracture surface, the location of fatigue crack initiation and its propagation behavior were clarified. The results obtained are summarized as follows.

A hysteresis loop is formed in the loading and unloading processes, and superelasticity that recovers the strain upon unloading appears after USP. The value of the upper stress plateau under the loading process is increased by USP. The increase in stress is almost proportional to the USP coverage.

In the rotating-bending fatigue test at room temperature in air, the fatigue life is improved by USP treatment with coverages of 200,1000 , and $2000 \%$, but the fatigue life is almost equal to that of the as-received material at the coverage of $4000 \%$. The higher the coverage, the higher the percentage of fatigue cracks in USP-treated specimens, which occur from the specimen surface. 


\section{Acknowledgments}

The experimental work in this study was carried out with the assistance of students of Aichi Institute of Technology, to whom the author wishes to express his gratitude. The author also expresses his deep gratitude to Dr. Kanehisa Hattori (Toyo Seiko Co., Ltd., Japan) for the experimental help in the X-ray diffraction measurement.

\section{References}

1 K. Otsuka and C. M. Wayman: Shape Memory Materials, K. Otsuka and C. M. Wayman, Eds. (Cambridge University Press, Cambridge, 1998) Chap. 1.

2 Y. Suzuki and Y. Sekiguchi: Shape Memory Alloys, H. Funakubo, Ed. (Gordon and Breach Science Publishers, New York, 1987) Chap. 3.

3 H. Cho, Y. Takeda, and T. Sakuma: Advances in Shape Memory Materials, Q. P. Sun, R. Matsui, K. Takeda, and E. A. Pieczyska, Eds. (Springer, Cham, 2017) pp. 1-15. https://doi.org/10.1007/978-3-319-53306-3

4 W. M. Huang, X. Y. Gao, B. K. Loo, L. M. He, and B. K. A. Ngoi: Proc. SMST-SMM2001 Conf. Shape Memory and Superelastic Technologies and Shape Memory Materials (Trans Tech Publications, 2002) 87-90.

5 H. Tobushi, K. Mitsui, and K. Takeda: J. Theor. Appl. Mech. 50 (2012) 855. http://www.ptmts.org.pl/jtam/ index.php/jtam/article/view/v50n3p855/147

6 K. N. Melton: Ni-Ti Based Shape Memory Alloys: Engineering Aspects of Shape Memory Alloys, T. W. Duerig, K. N. Melton, D. Stockel, and C. M. Wayman, Eds. (Butterworth-Heinemann, London, 1990) Part 1, pp. 21-35.

7 R. Matsui, K. Takeda, H. Tobushi, and E. A. Pieczyska: J. Theor. Appl. Mech. 56 (2018) 447. https://doi. org/10.15632/jtam-pl.56.2.447

8 K. Yamada and R. Matsui: Key Eng. Mater. 725 (2017) 389. https://doi.org/10.4028/www.scientific.net/ KEM.725.389

9 P. H. Lin, H. Tobushi, T. Hashimoto, Y. Shimeno, and K. Takata: Mater. Sci. Res. Int. 7 (2001) 103.

10 R. Matsui, H. Tobushi, Y. Furuichi, and H. Horikawa: Trans. ASME 126 (2004) 384. https://doi. org/10.1115/1.1789952

11 T. Sawaguchi, G. Kaustrater, A. Yawny, M. Wagner, and G. Eggeler: Metall. Mater. Trans. A 34 (2003) 2847. https://doi.org/10.1007/s11661-003-0186-X

12 R. Matsui, Y. Makino, H. Tobushi, Y. Furuichi, and F. Yoshida: Mater. Trans. 47 (2006) 759. https://doi. org/10.2320/matertrans.47.759

13 K. Takeda, R. Matsui, H. Tobushi, S. Homma, and K. Hattori: Mater. Trans. 56 (2015) 513. https://doi. org/10.2320/matertrans.MBW201408

14 S. K. Cheong, D. S. Lee, J. H. Lee, M. Handa, and Y. Watanabe: Proc. ICSP-10 (2008) 494-498.

15 J. E. Shigley and C. R. Mischke: Mechanical Engineering Design (McGraw-Hill, New York, 1989) 5th ed., pp. 282-283.

\section{About the Author}

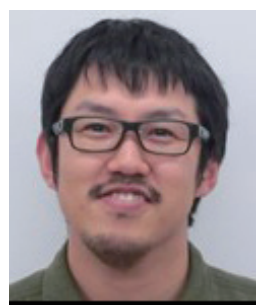

Kohei Takeda received his B.S., M.S., and Ph.D. degrees from Aichi Institute of Technology, Japan, in 2008, 2011, and 2014, respectively. Since 2015, he has been an assistant professor at Aichi Institute of Technology. His research interests are in surface modification, intelligent materials, smart actuators, and additive manufacturing. (k-takeda@aitech.ac.jp) 